ARTICLE

\title{
Quantum tunnelling and charge accumulation in organic ferroelectric memory diodes
}

Matteo Ghittorelli ${ }^{1, \star}$, Thomas Lenz ${ }^{2,3, \star}$, Hamed Sharifi Dehsari ${ }^{2}$, Dong Zhao ${ }^{2}$, Kamal Asadi $^{2}$, Paul W.M. Blom ${ }^{2}$, Zsolt M. Kovács-Vajna1, Dago M. de Leeuw ${ }^{2,4} \&$ Fabrizio Torricelli ${ }^{1}$

Non-volatile memories-providing the information storage functionality-are crucial circuit components. Solution-processed organic ferroelectric memory diodes are the non-volatile memory candidate for flexible electronics, as witnessed by the industrial demonstration of a $1 \mathrm{kbit}$ reconfigurable memory fabricated on a plastic foil. Further progress, however, is limited owing to the lack of understanding of the device physics, which is required for the technological implementation of high-density arrays. Here we show that ferroelectric diodes operate as vertical field-effect transistors at the pinch-off. The tunnelling injection and charge accumulation are the fundamental mechanisms governing the device operation. Surprisingly, thermionic emission can be disregarded and the on-state current is not space charge limited. The proposed model explains and unifies a wide range of experiments, provides important design rules for the implementation of organic ferroelectric memory diodes and predicts an

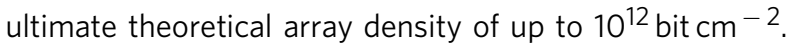

\footnotetext{
${ }^{1}$ Department of Information Engineering, University of Brescia, Via Branze 38, 25123 Brescia, Italy. ${ }^{2}$ Max Planck Institute for Polymer Research, Ackermannweg 10, 55128 Mainz, Germany. ${ }^{3}$ Graduate School Materials Science in Mainz, Staudinger Weg 9, 55128 Mainz, Germany. ${ }^{4}$ Faculty of Aerospace Engineering, Delft University of Technology, Kluyverweg 1, 2629 HS Delft, The Netherlands. ${ }^{\star}$ These authors contributed equally to this work. Correspondence and requests for materials should be addressed to F.T. (email: fabrizio.torricelli@unibs.it).
} 
$\mathrm{N}$ on-volatile memories-providing the information storage functionality-are crucial circuit components, finding application in several fields such as health care, wellness, communication, automotive, entertainment, consumer electronics, and so on ${ }^{1-3}$. Solution-processed organic non-volatile memory diodes based on ferroelectric and semiconducting polymers are recognized as the non-volatile memory candidate for flexible electronics ${ }^{4}$. Organic ferroelectric memory diodes comprise blends of a semiconducting polymer and the ferroelectric copolymer of vinylidenefluoride with trifluoroethylene $(\mathrm{P}(\mathrm{VDF}-\mathrm{TrFE}))^{5-18}$. Upon film formation, the blend decomposes by spinodal phase separation yielding a microstructure that consists of bicontinuous columnar domains of the semiconducting polymer embedded in the matrix of P(VDF$\operatorname{TrFE})^{12,13,16,19}$. In the memory diode the blend film is sandwiched between two electrodes. The injecting electrode is deliberately chosen such that it forms a high-barrier Schottky contact with the semiconductor. Hence, the charge transport is injection limited and the current density is low; the diode is in the off-state. When the ferroelectric polymer is fully polarized, the current density is high and the diode is in the on-state. The diode can reversibly be switched by reversing the ferroelectric polarization. A 9 bit $(3 \times 3)$ cross-bar memory array was demonstrated in 2010 for the first time $e^{20}$. State of the art is the industrial demonstration of a $1 \mathrm{kbit}$ reconfigurable array fabricated on plastic foil ${ }^{4}$.

Further progress, however, is limited owing to the lack of understanding of the device physics, which is required for the technological implementation of high-density arrays. Originally, it was suggested that the ferroelectric polarization leads to band bending in the semiconductor at the injecting contact $5,6,18,21,22$. This explanation however required a particular three-dimensional morphology showing an undercut of the ferroelectric slab, which was not observed in thorough morphological analyses ${ }^{12,13,16,19}$. In fact, the driving force for resistive switching is the modulation of the injection barrier by the ferroelectric polarization. Later, it was proposed, basing on numerical simulations, that the origin of the barrier lowering is the stray electric field between the polarization charges of the ferroelectric polymer and the compensating image charges in the electrode ${ }^{8,9}$. The ferroelectric polarization has been artificially modelled by means of fixed charges located at $1.5 \mathrm{~nm}$ distance from the electrode. The calculated current modulation was rationalized as a function of injection barrier. However, the complete current-voltage $(I-V)$ curves could not be modelled because the polarization was simply described by fixed charges, disregarding its dependence on the electric field.

Here, we perform two-dimensional (2D) numerical simulations including the $2 \mathrm{D}$ polarization of the ferroelectric polymer, the charge injection at the metal-semiconductor interface and the charge transport in the organic semiconductor. We show that the full $I-V$ characteristics as a function of both bias and temperature can be quantitatively modelled. The simulations reveal the crucial role of lateral ferroelectric polarization on the charge transport. The charge injection is analysed and quantum tunnelling is identified as the dominant mechanism. Finally, the interfacial charge transport and the consequences for downscaling are discussed.

\section{Results}

Structure and electrical characteristics of memory diodes. Phase separated blend memory diodes were fabricated using the ferroelectric polymer $\mathrm{P}(\mathrm{VDF}-\mathrm{TrFE})$ and the semiconducting polymer poly(9,9-dioctylfluorene) (PFO) by means of wire-bar coating, according to the method proposed in ref. 12. Further details are reported in the Methods section. The device area amounted to $0.16 \mathrm{~mm}^{2}$. An atomic force microscopy (AFM) height image is presented in Fig. 1a. The microstructure exhibits the characteristic morphology of a phase separated blend and consists of bicontinuous columnar PFO domains embedded in a $\mathrm{P}(\mathrm{VDF}-\mathrm{TrFE})$ matrix $^{12}$. The needle-like morphology is typical for semicrystalline $\mathrm{P}(\mathrm{VDF}-\mathrm{TrFE})^{23,24}$. The schematic cross-section of a memory diode is shown in Fig. 1b. This microstructure is used as device geometry for $2 \mathrm{D}$ numerical simulations. The $I-V$ characteristics measured at ambient temperature in dynamic vacuum of $10^{-6}$ mbar are presented in Fig. 1c (symbols). The top PEDOT:PSS contact is grounded and we sweep the bottom $\mathrm{Au}$ electrode from 0 to $+20 \mathrm{~V}$ and back. We start in the off-state. At low bias the current density is low as the charge injection at the Au-PFO contact is limited. By increasing the positive bias, the ferroelectric $\mathrm{P}(\mathrm{VDF}-\mathrm{TrFE})$ gets fully polarized above the coercive voltage, here about $10 \mathrm{~V}$. The current then increases by orders of magnitude. Upon sweeping back to $0 \mathrm{~V}$, the ferroelectric polarization does not change and the diode remains in the on-state. To switch off the diode, the ferroelectric polarization has to be reversed by applying a negative voltage larger than the coercive voltage.

Numerical model. To explain the operation of ferroelectric memory diodes we reproduced the measured electrical characteristics as a function of temperature by means of $2 \mathrm{D}$ numerical simulations. The charge transport in the organic semiconductor is described by means of the variable range hopping theory ${ }^{25}$ and accounting for the disorder ${ }^{26-28}$. A Gaussian density of states centred at the Highest Occupied Molecular Orbital (HOMO) level of PFO is assumed ${ }^{29-31}$. The Poisson, continuity and drift-diffusion transport equations are solved on a $2 \mathrm{D}$ grid $^{32-35}$. The charge flow at the metal-semiconductor contact is described accounting for the drift-diffusion, energy disorder, thermionic emission, tunnelling and image force barrier lowering ${ }^{36-40}$. The numerical simulations account for the $2 \mathrm{D}$ energy barrier, electric field and charge concentration. We emphasize that the polarization of the ferroelectric polymer as a function of the electric field is explicitly taken into account ${ }^{34,41,42}$. The charge flow at the metal-semiconductor contacts, the charge transport in the semiconductor, and the ferroelectric polarization are solved together on a $2 \mathrm{D}$ grid that accounts for the device geometry. The numerical framework and the physical model are detailed in the Supplementary Notes 1-4.

The model parameters are the following. The transport parameters of the semiconductor, namely the average intersite distance $d_{\mathrm{a}}=1.5 \mathrm{~nm}$ and the energy disorder $\sigma=0.16 \mathrm{eV}$, are obtained by modelling the temperature-dependent measurements of a PFO hole-only diode with Ohmic contacts (Supplementary Fig. 1). The HOMO level of $\mathrm{PFO}$ is taken as $E_{\mathrm{HOMO}}=5.8 \mathrm{eV}$ (refs 43,44). Since the $\mathrm{Au}$ work function is taken as $4.5 \mathrm{eV}$ (refs 45,46), the contact barrier is $\Phi_{\mathrm{B} 0}=1.3 \mathrm{eV}$ (ref. 6). We extracted the ferroelectric parameters of $\mathrm{P}(\mathrm{VDF}-\mathrm{TrFE})$, namely the relative permittivity, the remanent polarization, the saturation polarization and the coercive field as a function of temperature from thin film capacitor measurements (Supplementary Fig. 2). All the model parameters are listed in Supplementary Table 1. The diode thickness is $265 \pm 10 \mathrm{~nm}$ and the areal density of PFO pillars is estimated from AFM topography analysis. Since the diameter of the PFO pillars randomly varies along the area of the device, we measured the topography on several spots and we found that the average $\mathrm{PFO} / \mathrm{P}(\mathrm{VDF}-\mathrm{TrFE})$ interface length is $16.8 \pm 2.3 \mu \mathrm{m}$ on an area of $5 \times 5 \mu \mathrm{m}^{2}$, as shown in Supplementary Note 5 . The measured and calculated $I-V$ characteristics at ambient temperature are shown on a semilogarithmic scale in Fig. 1c. The inset shows the $I-V$ characteristics on a linear scale. The temperature-dependent curves are presented in 
a

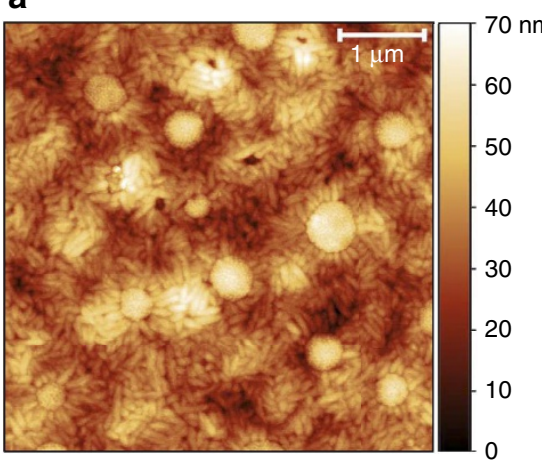

b

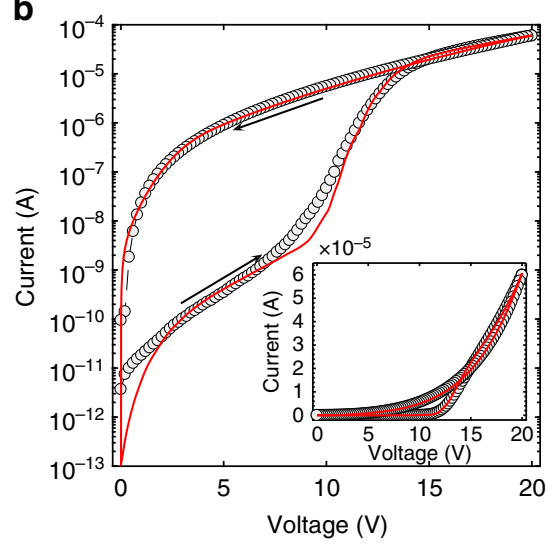

d

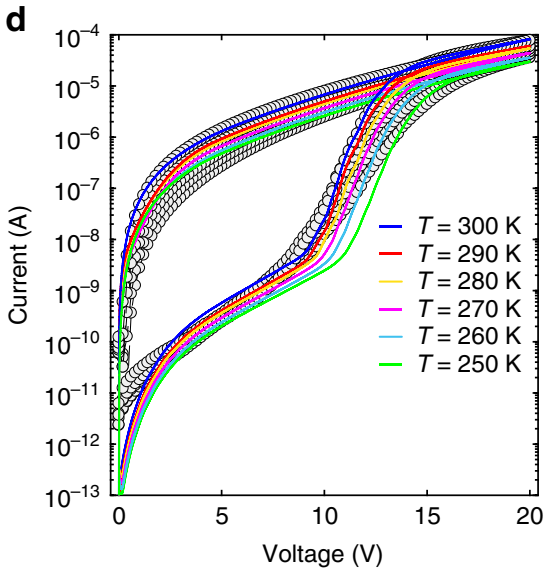

C

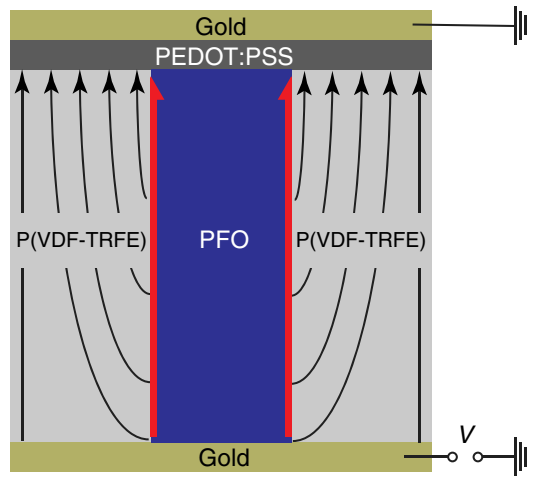

Figure 1 | Structure and characteristics of organic ferroelectric memory diodes. (a) AFM micrograph of a phase-separated blend film of PFO and P(VDF-TrFE). The film thickness amounted to $265 \pm 10 \mathrm{~nm}$. (b) Schematic cross-section of a ferroelectric memory diode. For the sake of clarity, here only one pillar of PFO surrounded by P(VDF-TrFE) is shown. The black arrows indicate the 2D polarization of the ferroelectric polymer. Holes are efficiently transported at the PFO/P(VDF-TRFE) interface (red arrows). (c) Measured (symbols) and simulated (line) I-V characteristics at ambient temperature $(290 \mathrm{~K})$. (d) Measured (symbols) and simulated (lines) I-V characteristics as a function of temperature.

Fig. 1d. The simulations nicely predict both the on-state and the off-state current in the whole range of biases and temperatures.

Operation of memory diodes. The $2 \mathrm{D}$ numerical simulations provide physical insight into the device operation. By increasing the applied voltage, the ferroelectric polymer polarizes and, owing to the stray field, the charge carriers are efficiently injected into the semiconductor. Figure $2 \mathrm{a}$ shows the distribution of the charge density in the PFO pillar when biased at $20 \mathrm{~V}$. The injected charge carriers are located at the ferroelectric-semiconductor interfaces along the whole thickness of the diode. Two separated 'channels' are formed. Figure $2 \mathrm{~b}$ is a zoom of Fig. $2 \mathrm{a}$ at the right $\mathrm{PFO} / \mathrm{P}(\mathrm{VDF}-\mathrm{TrFE})$ interface. The charge concentration at the interface is $2 \times 10^{19} \mathrm{~cm}^{-3}$, it is $\sim 10^{18} \mathrm{~cm}^{-3}$ at a distance of $2.5 \mathrm{~nm}$ from the interface, and it drops to $10^{15} \mathrm{~cm}^{-3}$ in the centre of the PFO pillar. It is worth noting that in the centre of the PFO pillar the large energy barrier at the injecting contact depletes the semiconductor over the whole diode thickness. The charge carrier density in the accumulation layer is four orders of magnitude higher than that in the centre of the pillar. The origin of the accumulation is the lateral $x$-component of the polarization vector in the $\mathrm{P}(\mathrm{VDF}-\mathrm{TrFE})$ slab.

The role of the $x$-polarization. Based on the diode geometry, it is expected that the polarization is oriented along the $y$-direction. Hence, the formation of the $x$-polarization component is counterintuitive and it can be explained as follows. The large stray electric field between the polarization charges of the ferroelectric polymer and the compensating image charges in the electrode lowers the barrier, and enables the efficient charge injection at the bottom corner of the semiconductor-ferroelectric interface. The injected carriers set the local potential into the semiconductor equal to the bottom contact potential (Fig. $2 \mathrm{c}, x=265 \mathrm{~nm}$ ), the electric field lines bend and a lateral $x$-component of the electric field $\left(F_{x}\right)$ rises. Consequently, the electric field lines are no longer parallel to the interface. When $F_{x}$ is larger than the coercive field, a $P_{x}$ polarization component is created and the displacement $\left(D_{x}\right)$ is enhanced. $D_{x}$ is compensated by charge carriers, which further accumulate at the PFO/P(VDF-TrFE) interface. An accumulated channel along the whole interface from the bottom to the top contact is formed. The hole density evolution as a function of the applied voltage is discussed in Supplementary Note 6.

Figure $2 \mathrm{~d}$ shows the $x$-polarization distribution in the $\mathrm{P}(\mathrm{VDF}-\mathrm{TrFE})$ domains. The $x$-polarization is about $0.3 \mu \mathrm{Ccm}^{-2}$. Although the $x$-polarization is one order of magnitude lower than the $y$-polarization, it is high enough to maintain the charge accumulation. When the applied voltage is reduced, the $x$-polarization is retained by the ferroelectric polymer and, hence, the accumulated channel at the interface is preserved. This is confirmed by Supplementary Fig. 10, where the hole density at the right $\mathrm{PFO} / \mathrm{P}(\mathrm{VDF}-\mathrm{TrFE})$ interface with $V=5 \mathrm{~V}$ is shown. Also in this case, a channel is accumulated. The $x$-polarization is perfectly symmetric around the centre of the PFO pillar, meaning that the negative polarization vectors at the left interface are 
a

b

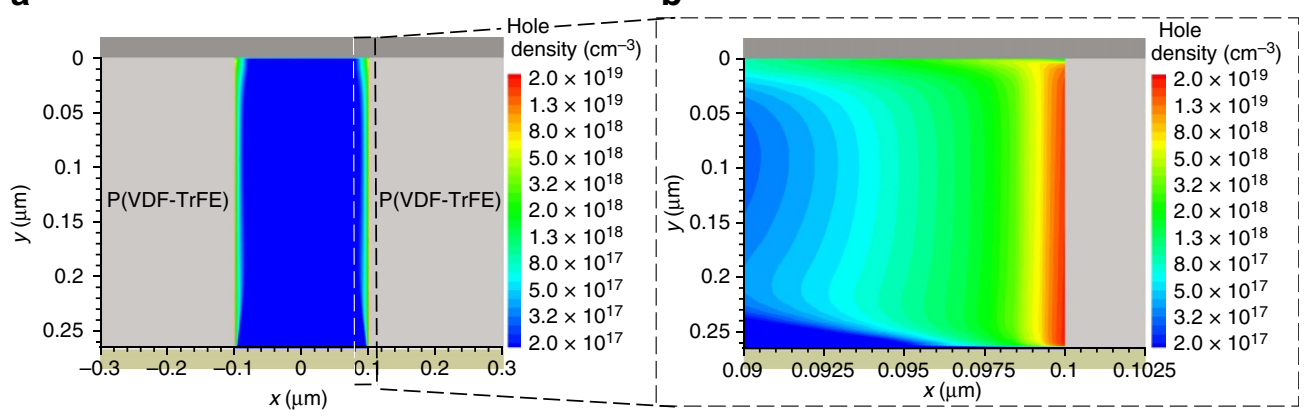

C
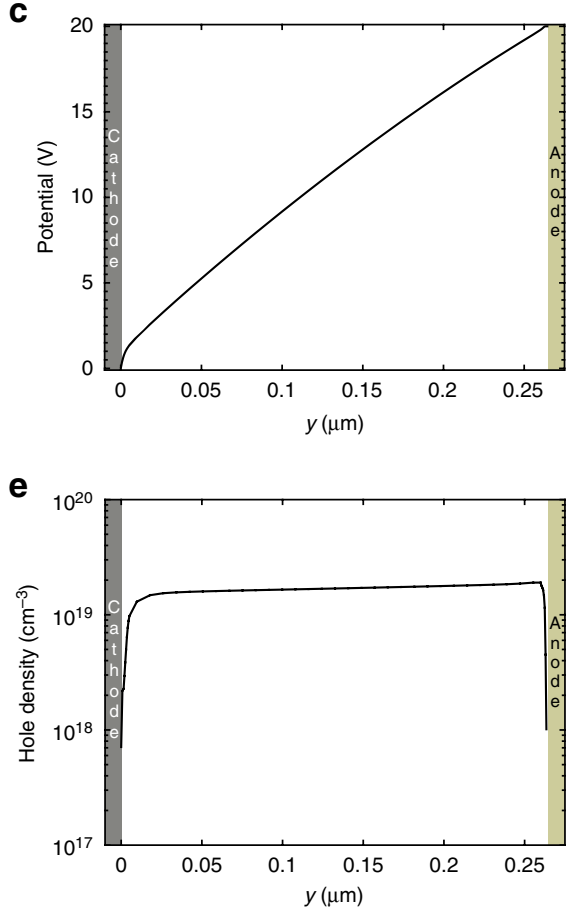

d

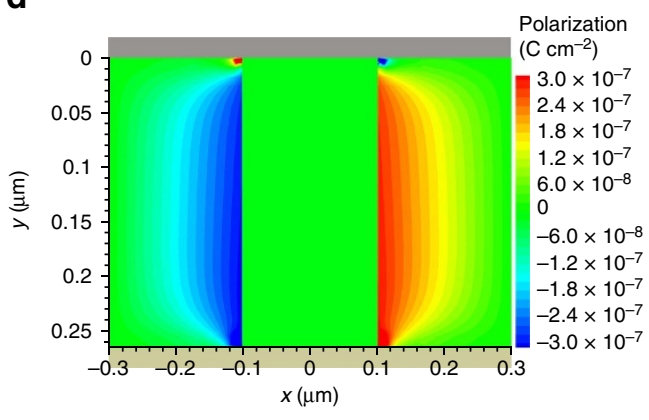

f

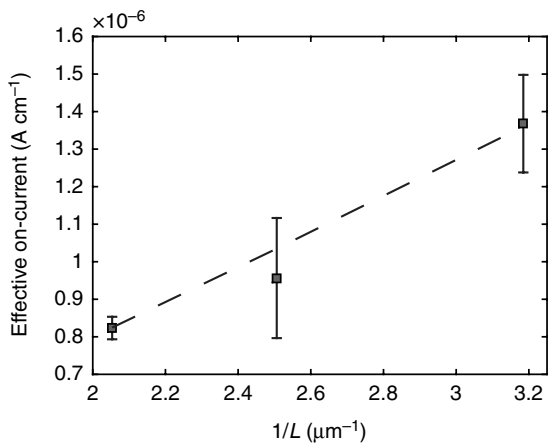

Figure 2 | Operation of organic ferroelectric memory diodes. (a) Hole density distribution in the PFO pillar at $V=20 \mathrm{~V}$. (b) Zoom of the hole density distribution at the right PFO/P(VDF-TrFE) interface. (c) Potential along the PFO/P(VDF-TrFE) interface (cut line at $x=0.099 \mu \mathrm{m}$ ), $V=20 \mathrm{~V}$. The anode corresponds to the injecting contact, while the cathode corresponds to the collecting contact. (d) $x$-component of the polarization vector at $V=20 \mathrm{~V}$. (e) Hole density along the PFO/P(VDF-TrFE) interface (cut line at $x=0.099 \mu \mathrm{m}$ ), $\mathrm{V}=20 \mathrm{~V}$. (f) Effective (viz. interface length normalized) current at $V=10 \mathrm{~V}$ as a function of the inverse of the device thickness $(L)$. The dashed line is the linear least-square approximation. The error bars are the s.d.

mirrored by the positive polarization vectors at the right interface (further details are reported in Supplementary Note 7). The $x$-polarization is maximum (minimum) at right (left) side of the injecting bottom contact because of the stray field. The reverse situation holds at the collecting top contact because the stray field points in the opposite direction. Therefore, close to the extracting contact the charge carriers are pushed away from the interface. This results in a pinch-off of the accumulated channel.

Scaling of the diode thickness. As a confirmation, Fig. 2e shows the charge carrier density $(p)$ along the $\mathrm{PFO} / \mathrm{P}(\mathrm{VDF}-\mathrm{TrFE})$ interface between the two electrodes. The injecting bottom contact is located at $y=265 \mathrm{~nm}$, while the extracting top contact is located at $y=0 \mathrm{~nm}$. The injecting contact shows a $3 \mathrm{~nm}$ depletion region because of the width of the energy barrier at the contact, lowered by the stray field. The hole density is almost constant along the $\mathrm{PFO} / \mathrm{P}(\mathrm{VDF}-\mathrm{TrFE})$ interface. This situation is completely different from a space charge-limited diode, where the charge concentration decreases with the square root of the distance from the injecting contact $(p \sim 1 / \sqrt{y})^{30}$ and the resulting current is described by the Mott-Gurney law ${ }^{47}$, which reads $I \sim V^{2} / L^{3}$, where $L$ is the layer thickness. At the collecting top contact the charge concentration drops down to $10^{17} \mathrm{~cm}^{-3}$. The drop of the hole density is due to the stray field at the top contact that points in the opposite direction with respect to the injecting bottom contact (Fig. 2d). The hole density profile resembles that of a field-effect transistor operating in the saturation regime, where the drain current is proportional to $I \sim V^{2} / L, V=V_{\mathrm{G}}-V_{\mathrm{T}}$, where $V_{\mathrm{G}}$ is the gate voltage and $V_{\mathrm{T}}$ is the threshold voltage ${ }^{48}$. In the memory diode the bottom contact is the source, the top contact is the drain and the gate voltage is equivalent to the drain voltage. This gives by definition a current proportional to $V^{2}$. Although this is the same mathematical $I-V$ relation as given by the Mott-Gurney law, the underlying physics is completely different. This can be further demonstrated by scaling the thickness $L$ of the fabricated memory diodes (details in Supplementary Note 8). Figure $2 \mathrm{f}$ shows the measured on-current as a function of $1 / L$ at $V=10 \mathrm{~V}$. The current linearly depends on 
the inverse of the thickness, and thus $I \sim V^{2} / L$ as in field-effect transistors operating at pinch-off.

Charge injection mechanism. Figure 3 a shows the charge carrier concentration in the PFO pillar close to the $\mathrm{P}(\mathrm{VDF}-\mathrm{TrFE})$ domain and the injecting electrode. The depletion width due to the contact barrier is less than $3 \mathrm{~nm}$, which is compatible with tunnelling charge injection. To elucidate the contribution of the tunnelling on the charge injection current, Fig. $3 \mathrm{~b}$ shows the $I-V$ characteristics of a ferroelectric memory diode calculated with and without tunnelling injection. The model without tunnelling (blue line) does not explain the measured current, whereas the model including tunnelling charge injection (red line) perfectly describes the experimental data in both on- and off-states. A a
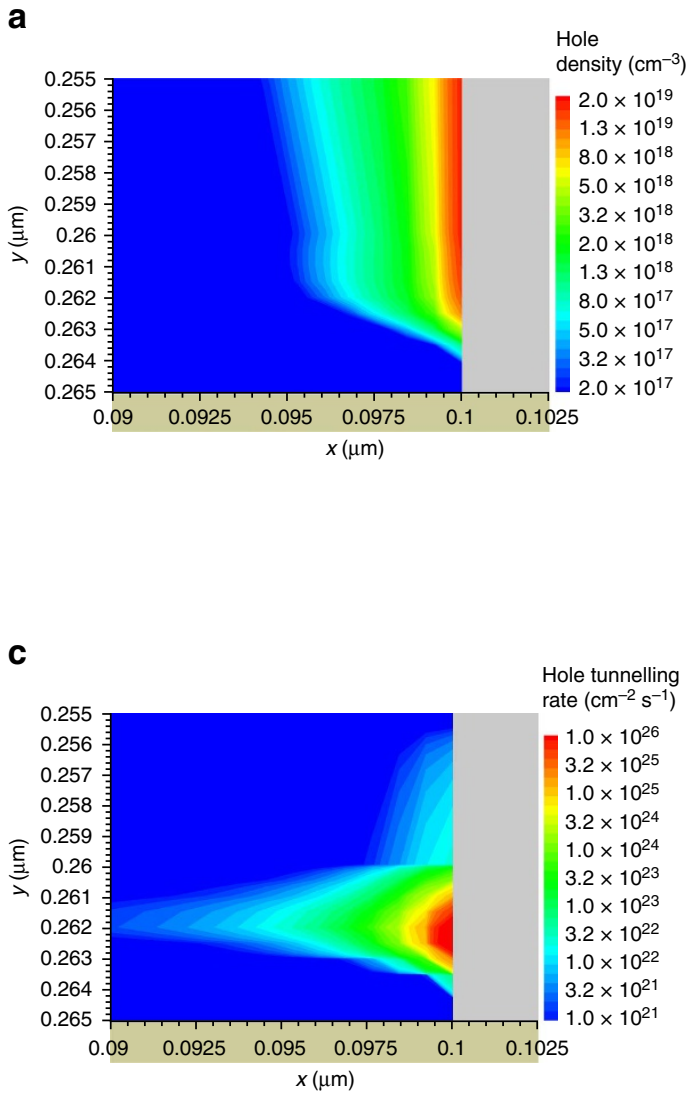

e

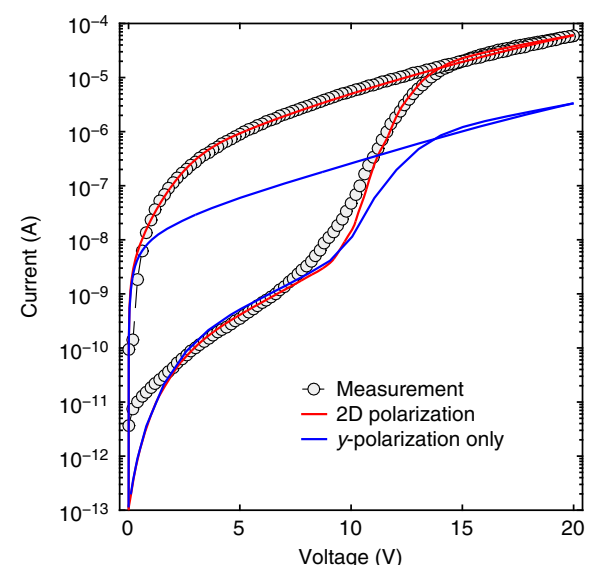

b

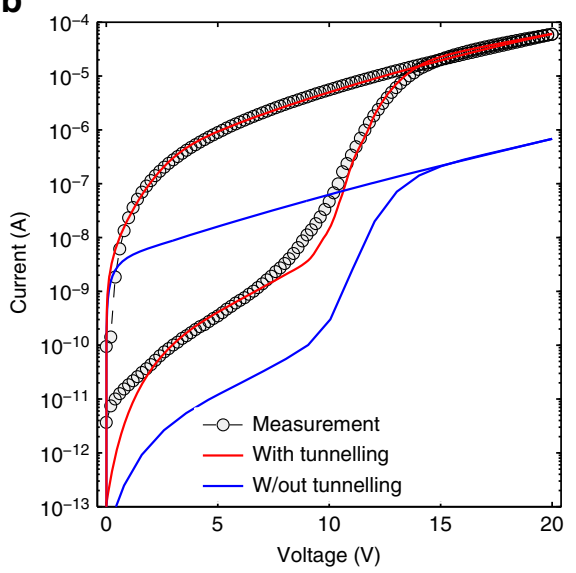

d

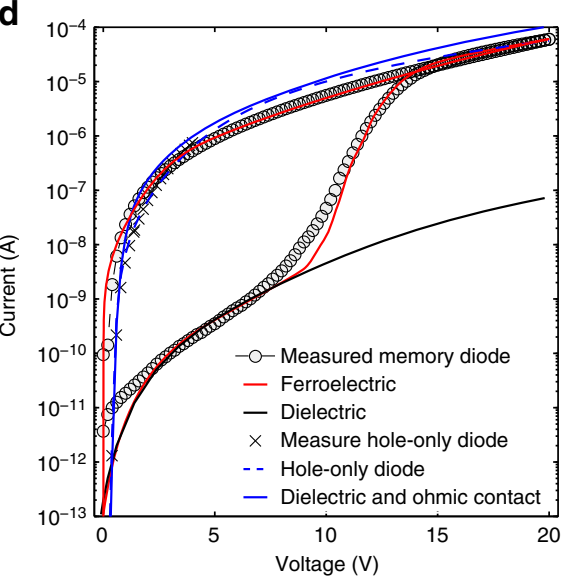

f

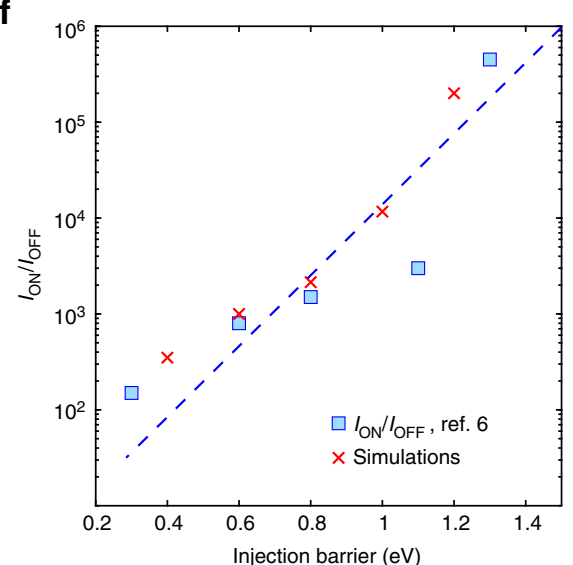

Figure 3 | Injection and polarization in organic ferroelectric memory diodes. (a) Hole density distribution within the PFO at the interface-contact corner, $V=20 \mathrm{~V}$. (b) Measured (symbols) and simulated (lines) I-V characteristics at ambient temperature. The blue line is calculated by switching off the tunnelling. (c) Tunnelling rate within the PFO pillar at the interface-contact corner, $V=20 \mathrm{~V}$. (d) $I-V$ characteristics calculated by varying the contact barrier and the ferroelectric properties. (e) $I-V$ characteristics calculated accounting for the $2 \mathrm{D}$ polarization $\left(P_{x}+P_{y}\right.$, red line) and only $y$-component $P_{y}$ (blue line). (f) On/off current ratio as a function of injection barrier. Symbols correspond to experimental data taken from ref. 6, while the red crosses are the result of the simulations, and are obtained at $F_{y}=2 \times 10^{5} \mathrm{~V} \mathrm{~cm}^{-1}$. The dashed line is the least-square approximation of the data taken from ref. 6 . The slope is $0.25 \mathrm{eV} \mathrm{dec}^{-1}$. 
a

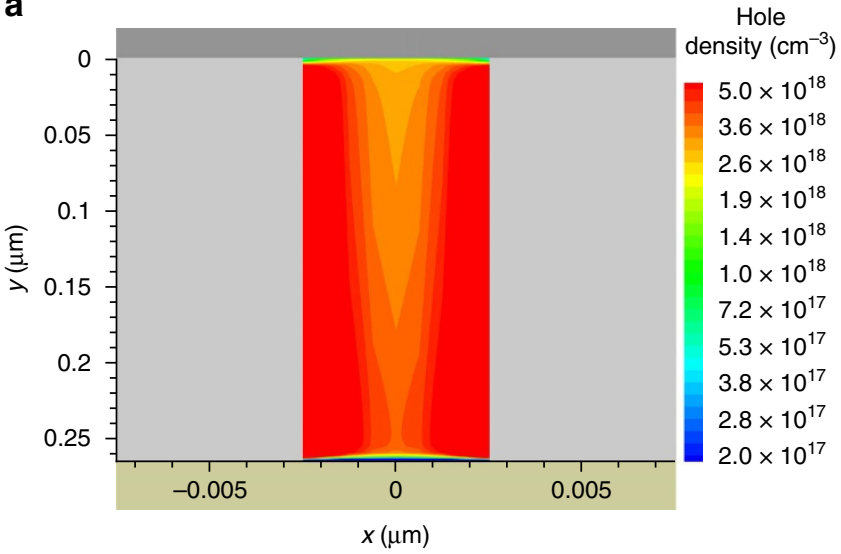

b

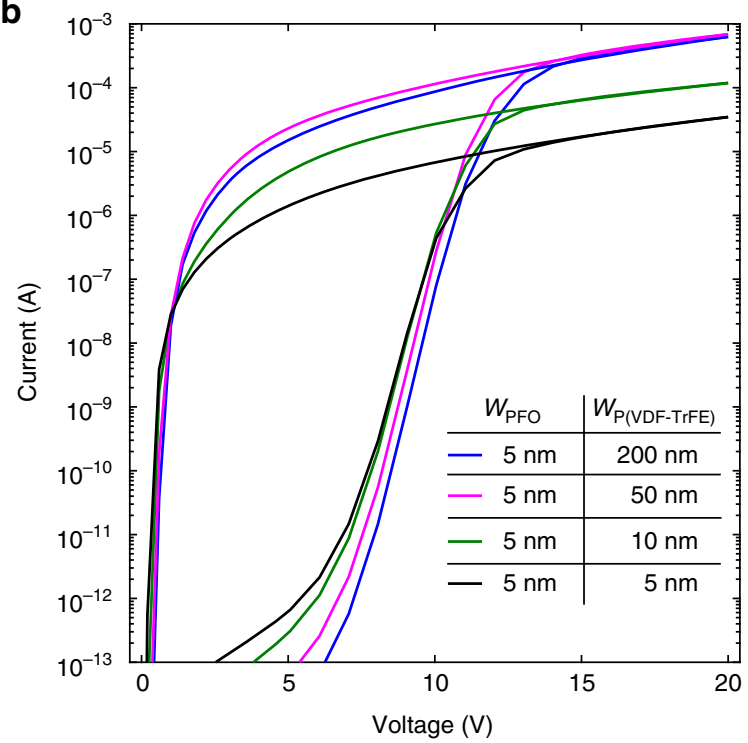

Figure 4 | Scaling of organic ferroelectric memory diodes. (a) Hole concentration in the PFO pillar with lateral dimension $W=W_{\mathrm{PFO}}$ $+W_{\mathrm{P} \text { (VDF-TrFE) }}$, where $W_{\mathrm{PFO}}=5 \mathrm{~nm}$ and $W_{\mathrm{P} \text { (VDF-TrFE })}=200 \mathrm{~nm}$, biased at $5 \mathrm{~V}$. (b) Comparison between the calculated $I-V$ characteristics as a function of the $P(V D F-T r F E)$ lateral dimension $\left(W_{P(V D F-T r F E}\right)$ with $W_{P F O}=5 n$.

detailed analysis is shown in Supplementary Note 9. It is surprising that the thermionic emission can be disregarded with respect to the tunnelling. Figure $3 c$ shows the hole tunnelling rate at the injecting contact. The tunnelling rate is as high as $10^{26} \mathrm{~cm}^{-2} \mathrm{~s}^{-1}$, efficiently providing all the charges transported in the accumulated channel. The width of the energy barrier is only $2.5 \mathrm{~nm}$. The ferroelectric polymer is fully polarized and the tunnelling is due to the stray field (high local electric field) and to the accumulated channel (small depletion width). The metal-semiconductor Schottky contact behaves like an ideal Ohmic contact.

\section{Discussion}

The ferroelectric polarization not only modulates the injection barrier but also yields a very high on-state current. In Fig. $3 \mathrm{~d}$ we show the calculated $I-V$ characteristics by varying both the injection barrier and the polarization. First, the ferroelectric polymer is replaced with a linear dielectric by turning off the ferroelectric polarization. The current (black curve) is contact limited. Next, we set the energy barrier to zero $\left(\Phi_{\mathrm{B}}=0 \mathrm{eV}\right.$, blue full line) to obtain an ideal Ohmic contact. The resulting bulk-limited current is comparable to the current of a hole-only diode calculated by simply removing the $\mathrm{P}(\mathrm{VDF}-\mathrm{TrFE})$ slab (dashed blue line). The hole-only diode current is slightly lower since the dielectric causes a weak charge accumulation in the PFO pillar. By restoring the original energy barrier at the contact $\left(\Phi_{\mathrm{B}}=1.3 \mathrm{eV}\right)$ and switching on the ferroelectric polarization, the red curve is obtained. It perfectly reproduces the experimental data and surprisingly the memory diode current is comparable with that of the corresponding hole-only diode (viz. with equivalent semiconductor area as detailed in Supplementary Note 10). This can be explained as follows. In disordered organic semiconductors the mobility increases with the charge carrier density ${ }^{28,49}$. The increase of mobility combined with the strong hole accumulation compensates the reduction in the injection and transport area with respect to the corresponding bulk hole-only diode. This interpretation is confirmed by the weak temperature dependence of the on-state current. Figure 1d shows that on decreasing the temperature by $50 \mathrm{~K}$ the maximum on-current decreases only by a factor of 3 and the on-current at $3 \mathrm{~V}$ decreases by only one order of magnitude, which is reflected in low activation energies equal to 60 and $190 \mathrm{meV}$, respectively. The activation energy of hole-only diodes is typically much larger, between 200 and $600 \mathrm{meV}^{50}$.

The lateral $x$-polarization is crucial to obtain such high on-state current. Figure $3 \mathrm{e}$ shows the impact of the $x$-component of the polarization vector. The blue curve is calculated by considering only the $y$-component of the polarization vector $\left(\mathbf{P}=P_{y}\right)$. The current is more than one order of magnitude lower than that obtained with the $2 \mathrm{D}$ polarization vector $\left(\mathbf{P}=P_{x}+P_{y}\right)$. The displacement given by the $x$-polarization is compensated by charges at the interface, which results in an accumulation layer. This already occurs in a linear dielectric where the displacement linearly depends on the electric field. However, in the ferroelectric domain the displacement is a nonlinear function of the electric field leading to an enhanced accumulation layer of only $2.5 \mathrm{~nm}$ (Fig. 2b). The shape of the $I-V$ characteristics is mainly defined by the linear response of the ferroelectric polymer to the electric field, while the magnitude of the on-current depends on the $x$-polarization. This is readily confirmed by Fig. 3e. The on-current calculated accounting for only the $P_{y}$ polarization has the same shape of that obtained accounting for both $P_{x}$ and $P_{y}$ polarization.

We calculate the on/off current ratio as a function of the injection barrier. The on/off current ratio is calculated at $5.3 \mathrm{~V}$, which corresponds to a vertical electric field $F_{Y}=2 \times 10^{5} \mathrm{~V} \mathrm{~cm}^{-1}$ to compare directly our results with ref. 6 . The on/off current ratio is shown on a semilogarithmic scale as a function of barrier in Fig. 3f. A linear relation is obtained with a slope of $0.27 \mathrm{eV} \mathrm{dec}^{-1}$, which perfectly agrees with the experimentally extracted value of $0.25 \mathrm{eV} \mathrm{dec}^{-1}$ (ref. 6). We note that the much lower slope of $0.067 \mathrm{eV} \mathrm{dec}^{-1}$ reported previously ${ }^{8}$ is due to the underestimated injection-limited current since only thermionic emission was taken into account. This again confirms that tunnelling is the dominant injection mechanism.

The strong confinement of both charge injection and charge transport shows that ferroelectric memory diodes are interface devices (Supplementary Note 11). We note that in previous reports current spreading within the semiconductor was calculated and, by considering only the dimension of the semiconductor slab, a minimum feature size of $50 \mathrm{~nm}$ was estimated ${ }^{8}$. Here we demonstrate that current spreading does not occur when the $2 \mathrm{D}$ polarization is taken into account. We found that both the charge injection and charge transport takes place in only $2.5-\mathrm{nm}$-thick channels. This enables the ultimate downscaling of the semiconductor lateral feature size down to $5 \mathrm{~nm}$. Figure 4a shows the charge carrier density in a PFO pillar of only $5 \mathrm{~nm}$ surrounded by slabs of P(VDF-TrFE) of $200 \mathrm{~nm}$. 
Although the applied bias is only $5 \mathrm{~V}$, which corresponds to the operating voltage of the diode, two accumulated channels occur and extend over the whole semiconductor thickness.

A comprehensive evaluation of the minimum lateral dimensions $(W)$ of a memory diode requires to consider the lateral dimensions of both the semiconductor $\left(W_{\mathrm{PFO}}\right)$ and the ferroelectric polymer $\left(W_{\mathrm{P}(\mathrm{VDF}-\mathrm{TrFE})}\right)$, i.e., $W=W_{\mathrm{PFO}}+W_{\mathrm{P}(\mathrm{VDF}-\mathrm{TrFE})}$. Therefore, we systematically varied $W_{\mathrm{P}(\mathrm{VDF}-\mathrm{TrFE})}$, while keeping $W_{\mathrm{PFO}}=5 \mathrm{~nm}$ constant; the device area is kept constant for the different cases, too. The resulting calculated $I-V$ characteristics are presented in Fig. $4 \mathrm{~b}$. The off-state current is similar in all cases. If $W_{\mathrm{P} \text { (VDF-TrFE) }}$ is reduced from $200 \mathrm{~nm}$ down to $5 \mathrm{~nm}$, the on-state current decreases. This is counterintuitive, as the decrease of the ferroelectric domain size results in an increase of the overall semiconductor area of the memory diode. Hence, a current increase is expected. However, the downscaling of $W_{\mathrm{P}(\mathrm{VDF}-\mathrm{TrFE})}$ leads to a strong decrease of the $x$-polarization component (details are shown in Supplementary Note 12) and, therefore, the charge accumulation is reduced. Although the charge carrier density in the channel decreases with decreasing $W_{\text {P(VDF-TrFE) }}$, Fig. $4 \mathrm{~b}$ shows that, even for the smallest feature size $W_{\mathrm{P}(\mathrm{VDF}-\mathrm{TrFE})}=5 \mathrm{~nm}$, a bistable diode is obtained with a current modulation of more than seven decades. This results in a maximum theoretical array density of about $10^{12} \mathrm{bit} \mathrm{cm}^{-2}$. Further discussions on device scaling and potential cross-talk between aggressively scaled devices are presented in the Supplementary Note 13.

In conclusion, we analyse the device physics of ferroelectric memory diodes based on a phase-separated blend of $\mathrm{P}(\mathrm{VDF}-\mathrm{TrFE})$ and PFO. We demonstrate that to understand the operational mechanism it is crucial to take into account the 2D ferroelectric polarization explicitly. The full $I-V$ characteristics are quantitatively described as a function of both bias and temperature. The key ingredient is the lateral polarization leading to the formation of a strongly accumulated hole density along the whole interface between the semiconducting and the ferroelectric polymer. Consequently, when the memory diode is operated in the on-state the current transport is not space charge limited but resembles the channel current in a field-effect transistor operated in saturation at pinch-off. This explains the $V^{2} / L$ dependence of the current, the low activation energy and the large current density in the on-state. The current modulation as a function of the energy barrier is quantitatively explained. The stray field modulates the barrier width but, surprisingly, thermionic emission can be disregarded. We discover that in ferroelectric memory diodes, where large contact barriers are required for large on/off current ratio, charge injection is dominated by tunnelling. Since there is no current spreading into the semiconductor, the feature size can ultimately be scaled down to $10 \mathrm{~nm}$, resulting in an ultimate theoretical array density of $10^{12} \mathrm{bit} \mathrm{cm}^{-2}$. The proposed model is general and can be applied for any combination of metal electrodes, semiconducting and ferroelectric polymers. The model leads to design rules for the implementation of ultrahigh-density organic non-volatile memories in several application fields including flexible displays, sensors, imperceptible and wearable electronics, biological and medical devices.

\section{Methods}

Device fabrication. The ferroelectric copolymer P(VDF-TrFE) with $65 \mathrm{~mol} \% \mathrm{VDF}$ and $35 \mathrm{~mol} \% \mathrm{TrFE}$ was purchased from Solvay. The number- and weight-average molecular weight, $M_{\mathrm{n}}$ and $M_{\mathrm{w}}$, were measured with gel permeation chromatography versus polystyrene standards and amounted to 147 and $296 \mathrm{~kg} \mathrm{~mol}^{-1}$, respectively. The polydispersity amounted to 2.01 . PFO, purchased from TNO/Holst Centre, has $M_{\mathrm{w}}=1,431 \mathrm{~kg} \mathrm{~mol}^{-1}$ and a polydispersity of 1.2 . The PEDOT:PSS (Clevios P VP Al 4083) was purchased from Heraeus.
$\mathrm{P}(\mathrm{VDF}-\mathrm{TrFE})$ and $\mathrm{PFO}$ were mixed in a ratio 9:1 by weight and then dissolved in cyclohexanone ( $45 \mathrm{mg}$ of the polymer mixture in $1 \mathrm{ml}$ cyclohexanone) at $80^{\circ} \mathrm{C}$ under vigorous stirring. Before use, the solution was filtered (Teflon filter with pore size of $1 \mu \mathrm{m})$. Fifty-nm-thick Au lines were thermally evaporated on cleaned glass slides (Schott Borofloat) through a shadow mask. Two-nm-thick $\mathrm{Cr}$ is deposited before $\mathrm{Au}$ as it serves as adhesion layer. Afterwards, the samples were treated with UV/ozone, before the films were deposited via wire-bar coating using K202 control coater (RK PrintCoat Instruments, UK). The plate of the wire-bar coater was heated to $80^{\circ} \mathrm{C}$. Subsequently, the samples were annealed for $2 \mathrm{~h}$ at $140^{\circ} \mathrm{C}$ in a vacuum oven to enhance the crystallinity of the ferroelectric P(VDF-TrFE). Film thickness was measured with a DEKTAK surface profilometer and amounted to $L=265 \mathrm{~nm}$.

Devices were fabricated in standard ambient conditions. To improve the yield, PEDOT:PSS was deposited by spin coating and used as top electrode ${ }^{34}$. The PEDOT:PSS top electrode was patterned by reactive ion etching and $50 \mathrm{~nm} \mathrm{Au}$ lines, which serves as self-aligned mask, and were evaporated through a shadow mask. Therefore, the sample was exposed to oxygen plasma (plasma technology) for $3 \mathrm{~min}$ at a pressure of $0.1 \mathrm{mbar}$. Each cross-point of the resulting crossbar array has an area of $\sim 0.16 \mathrm{~mm}^{2}$, which is the device area of a discrete memory diode.

Measurement set-up. AFM was performed in tapping mode using a Nanoscope Dimension 3100 (Bruker). Silicon tips with Al backside coating were used with a force constant of $26 \mathrm{~N} \mathrm{~m}^{-1}$ and a resonant frequency of $\sim 270 \mathrm{kHz}$. The electrical characteristics were acquired in vacuum at $10^{-6} \mathrm{mbar}$. The samples were fixed on a metal plate, which is connected to a cryostat (cooling with liquid $\mathrm{N}_{2}$ ) and to a temperature control unit (Oxford Intelligent Temperature Controller ITC 4). To ensure thermal contact, a layer of heat-conducting paste was applied between the plate and the substrate. Current-voltage characteristics were measured using a 4155B Semiconductor Parameter Analyser. The measurements were carried out only after the set temperature was stable for more than $10 \mathrm{~min}$.

Data availability. The data that support the findings of this study are available from the corresponding author on reasonable request.

\section{References}

1. Naber, R. C. G., Asadi, K., Blom, P. W. M., de Leeuw, D. M. \& de Boer, B. Organic nonvolatile memory devices based on ferroelectricity. Adv. Mater. 22, 933-945 (2010).

2. Heremans, P. et al. Polymer and organic nonvolatile memory devices. Chem. Mater. 23, 341-358 (2011)

3. Youn Jung, P., In-sung, B., Seok, Ju. K., Jiyoun, C. \& Cheolmin, P. Control of thin ferroelectric polymer films for non-volatile memory applications. IEEE Trans. Dielectr. Electr. Insul. 17, 1135-1163 (2010).

4. Van Breemen, A. J. J. M. et al. Crossbar arrays of nonvolatile, rewritable polymer ferroelectric diode memories on plastic substrates. Appl. Phys. Express 7, 031602 (2014)

5. Asadi, K., de Leeuw, D. M., de Boer, B. \& Blom, P. W. M. Organic non-volatile memories from ferroelectric phase-separated blends. Nat. Mater. 7, 547-550 (2008).

6. Asadi, K., de Boer, T. G., Blom, P. W. M. \& de Leeuw, D. M. Tunable injection barrier in organic resistive switches based on phase-separated ferroelectricsemiconductor blends. Adv. Funct. Mater. 19, 3173-3178 (2009).

7. Braz, T. et al. Morphology of ferroelectric/conjugated polymer phase-separated blends used in nonvolatile resistive memories. Direct evidence for a diffuse interface. J. Phys. Chem. C 119, 1391-1399 (2015).

8. Kemerink, M., Asadi, K., Blom, P. W. M. \& de Leeuw, D. M. The operational mechanism of ferroelectric-driven organic resistive switches. Org. Electron. 13, 147-152 (2012).

9. Khikhlovskyi, V. et al. Nanoscale organic ferroelectric resistive switches. J. Phys Chem. C 118, 3305-3312 (2014).

10. Khan, M. A., Bhansali, U. S., Cha, D. \& Alshareef, H. N. All-polymer bistable resistive memory device based on nanoscale phase-separated PCBMferroelectric blends. Adv. Funct. Mater. 23, 2145-2152 (2013).

11. Khikhlovskyi, V., van Breemen, A. J. J. M., Janssen, R. A. J., Gelinck, G. H. \& Kemerink, M. Data retention in organic ferroelectric resistive switches. Org. Electron. 31, 56-62 (2016)

12. Li, M. et al. Processing and low voltage switching of organic ferroelectric phase-separated bistable diodes. Adv. Funct. Mater. 22, 2750-2757 (2012).

13. McNeill, C. R., Asadi, K., Watts, B., Blom, P. W. M. \& de Leeuw, D. M. Structure of phase-separated ferroelectric/semiconducting polymer blends for organic non-volatile memories. Small 6, 508-512 (2010).

14. Michels, J. J., van Breemen, A. J. J. M., Usman, K. \& Gelinck, G. H. Liquid phase demixing in ferroelectric/semiconducting polymer blends: an experimental and theoretical study. J. Polym. Sci. Pol. Phys. 49, 1255-1262 (2011).

15. Su, G. M., Lim, E., Jacobs, A. R., Kramer, E. J. \& Chabinyc, M. L. Polymer side chain modification alters phase separation in ferroelectric-semiconductor polymer blends for organic memory. ACS Macro Lett. 3, 1244-1248 (2014) 
16. Su, G. M., Lim, E., Kramer, E. J. \& Chabinyc, M. L. Phase separated morphology of ferroelectric-semiconductor polymer blends probed by synchrotron X-ray methods. Macromolecules 48, 5861-5867 (2015).

17. van Breemen, A. et al. Surface directed phase separation of semiconductor ferroelectric polymer blends and their use in non-volatile memories. $A d v$. Funct. Mater. 25, 278-286 (2015).

18. Asadi, K., Wildeman, J., Blom, P. W. M. \& de Leeuw, D. M. Retention time and depolarization in organic nonvolatile memories based on ferroelectric semiconductor phase-separated blends. IEEE Trans. Electron Dev. 57, 3466-3471 (2010).

19. Asadi, K. et al. Spinodal decomposition of blends of semiconducting and ferroelectric polymers. Adv. Funct. Mater. 21, 1887-1894 (2011).

20. Asadi, K., Li, M., Stingelin, N., Blom, P. W. M. \& de Leeuw, D. M. Crossbar memory array of organic bistable rectifying diodes for nonvolatile data storage. Appl. Phys. Lett. 97, 193308 (2010).

21. Asadi, K., Blom, P. W. M. \& de Leeuw, D. M. The MEMOLED: active addressing with passive driving. Adv. Mater. 23, 865-868 (2011).

22. Sung, S. H. \& Boudouris, B. W. Systematic control of the nanostructure of semiconducting-ferroelectric polymer composites in thin film memory devices. ACS Macro Lett. 4, 293-297 (2015).

23. Lenz, T. et al. Microstructured organic ferroelectric thin film capacitors by solution micromolding. Phys. Stat Sol A 212, 2124-2132 (2015).

24. Katsouras, I. et al. The negative piezoelectric effect of the ferroelectric polymer poly(vinylidene fluoride). Nat. Mater. 15, 78-84 (2016).

25. Ambegaokar, V., Halperin, B. I. \& Langer, J. S. Hopping conductivity in disordered systems. Phys. Rev. B 4, 2612-2620 (1971).

26. Coehoorn, R., Pasveer, W. F., Bobbert, P. A. \& Michels, M. A. J. Charge-carrier concentration dependence of the hopping mobility in organic materials with Gaussian disorder. Phys. Rev. B 72, 155206 (2005).

27. Coehoorn, R. \& Bobbert, P. A. Effects of Gaussian disorder on charge carrier transport and recombination in organic semiconductors. Phys. Stat. Sol. A 209, 2354-2377 (2012).

28. Pasveer, W. F. et al. Unified description of charge-carrier mobilities in disordered semiconducting polymers. Phys. Rev. Lett. 94, 206601 (2005).

29. Bässler, H. Charge transport in disordered organic photoconductors a Monte Carlo simulation study. Phys. Stat. Sol. B 175, 15-56 (1993).

30. Kuik, M. et al. 25th Anniversary Article: charge transport and recombination in polymer light-emitting diodes. Adv. Mater. 26, 512-531 (2014).

31. Baranovskii, S. D. Theoretical description of charge transport in disordered organic semiconductors. Phys. Stat. Sol. B 251, 487-525 (2014).

32. Torricelli, F. et al. Ambipolar organic Tri-gate transistor for low-power complementary electronics. Adv. Mater. 28, 284-290 (2016).

33. Torricelli, F., Colalongo, L., Raiteri, D., Kovacs-Vajna, Zs. M. \& Cantatore, E. Ultra-high gain diffusion-driven organic transistor. Nat. Commun. 7, 10550 (2016).

34. Lenz, T. et al. Downscaling and charge transport in nanostructured ferroelectric memory diodes fabricated by solution micromolding. Adv. Funct. Mater. 26, 5111-5119 (2016).

35. Brondijk, J. J., Torricelli, F., Smits, E. C. P., Blom, P. W. M. \& de Leeuw, D. M. Gate-bias assisted charge injection in organic field-effect transistors. Org. Electron. 13, 1526-1531 (2012).

36. Emtage, P. R. \& O'Dwyer, J. J. Richardson-Schottky effect in insulators. Phys. Rev. Lett. 16, 356-358 (1966).

37. Ng, T. N., Silveira, W. R. \& Marohn, J. A. Dependence of charge injection on temperature, electric field, and energetic disorder in an organic semiconductor. Phys. Rev. Lett. 98, 066101 (2007).

38. Koehler, M., Biaggio, I. \& da Luz, M. G. E. Resolving the contact voltage dilemma in organic field effect transistors. Phys. Rev. B 78, 153312 (2008).

39. Silveira, W. R. \& Marohn, J. A. Microscopic view of charge injection in an organic semiconductor. Phys. Rev. Lett. 93, 116104 (2004).

40. van der Holst, J. J. M. et al. Modeling and analysis of the three-dimensional current density in sandwich-type single-carrier devices of disordered organic semiconductors. Phys. Rev. B 79, 085203 (2009).

41. Miller, S. L. \& McWhorter, P. J. Physics of the ferroelectric nonvolatile memory field effect transistor. J. Appl. Phys. 72, 5999-6010 (1992).

42. Miller, S. L., Nasby, R. D., Schwank, J. R., Rodgers, M. S. \& Dressendorfer, P. V. Device modeling of ferroelectric capacitors. J. Appl. Phys. 68, 6463-6471 (1990).

43. Janietz, S. et al. Electrochemical determination of the ionization potential and electron affinity of poly(9,9-dioctylfluorene). Appl. Phys. Lett. 73, 2453-2455 (1998).
44. Tengstedt, C. et al. Fermi-level pinning at conjugated polymer interfaces. Appl. Phys. Lett. 88, 053502 (2006).

45. Braun, S., Salaneck, W. R. \& Fahlman, M. Energy-level alignment at organic/metal and organic/organic interfaces. Adv. Mater. 21, 1450-1472 (2009).

46. Crivillers, N. et al. Large work function shift of gold induced by a novel perfluorinated azobenzene-based self-assembled monolayer. Adv. Mater. 25, 432-436 (2013)

47. Mott, N. F. \& Gurney, R. W. Electronics Process in Ionic Crystals (Oxford University Press, 1948)

48. Klauk, H. Organic thin-film transistors. Chem. Soc. Rev. 39, 2643-2666 (2010).

49. Tanase, C., Meijer, E. J., Blom, P. W. M. \& de Leeuw, D. M. Unification of the hole transport in polymeric field-effect transistors and light-emitting diodes. Phys. Rev. Lett. 91, 216601 (2003).

50. Craciun, N. I., Wildeman, J. \& Blom, P. W. M. Universal Arrhenius temperature activated charge transport in diodes from disordered organic semiconductors. Phys. Rev. Lett. 100, 056601 (2008).

\section{Acknowledgements}

We thank C. Kasparek for providing the temperature-dependent $I-V$ characteristics of the PFO hole-only diode. We gratefully acknowledge surface probe microscopy measurements by Dr Rüdiger Berger. We also hank Gunnar Glaßer for scanning electron microscopy measurements. We acknowledge the great MPIP technical support from C. Bauer, H. Burg, F. Keller and H. Raich. T.L. is grateful to the Graduate School Materials Science in Mainz for the financial support. K.A. is grateful to the Alexander von Humboldt Foundation for the funding that is provided in the framework of the Sofja Kovalevskaja Award, endowed by the Federal Ministry of Education and Research, Germany. F.T. acknowledges funding from the Fondazione Cariplo e Regione Lombardia, project 'New Opportunities and ways towards ERC' (NOW-ERC), no. 2014-2256. We acknowledge financial support from the Max Planck Institute for Polymer (MPIP) Research.

\section{Author contributions}

M.G., T.L., D.M.d.L. and F.T. conceived the idea and designed the experiments. M.G. performed the numerical simulations and the physical analysis. T.L. fabricated and measured the devices and contributed to the physical analysis. H.S.D. fabricated the devices. D.Z. performed the analysis of the ferroelectric capacitors. K.A., P.W.M.B. and Z.M.K.V. supervised the project. M.G., T.L., D.M.d.L. and F.T. wrote the paper. All the authors commented on the paper.

\section{Additional information}

Supplementary Information accompanies this paper at http://www.nature.com/ naturecommunications

Competing interests: The authors declare no competing financial interests.

Reprints and permission information is available online at http://npg.nature.com/ reprintsandpermissions/

How to cite this article: Ghittorelli, M. et al. Quantum tunnelling and charge accumulation in organic ferroelectric memory diodes. Nat. Commun. 8, 15841 doi: 10.1038/ncomms15841 (2017)

Publisher's note: Springer Nature remains neutral with regard to jurisdictional claims in published maps and institutional affiliations.

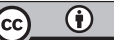

Open Access This article is licensed under a Creative Commons Attribution 4.0 International License, which permits use, sharing, adaptation, distribution and reproduction in any medium or format, as long as you give appropriate credit to the original author(s) and the source, provide a link to the Creative Commons license, and indicate if changes were made. The images or other third party material in this article are included in the article's Creative Commons license, unless indicated otherwise in a credit line to the material. If material is not included in the article's Creative Commons license and your intended use is not permitted by statutory regulation or exceeds the permitted use, you will need to obtain permission directly from the copyright holder. To view a copy of this license, visit http://creativecommons.org/ licenses/by/4.0/

(C) The Author(s) 2017 